\title{
Teachers' learning and assessing of mathematical processes with emphasis on representations, reasoning and proof
}

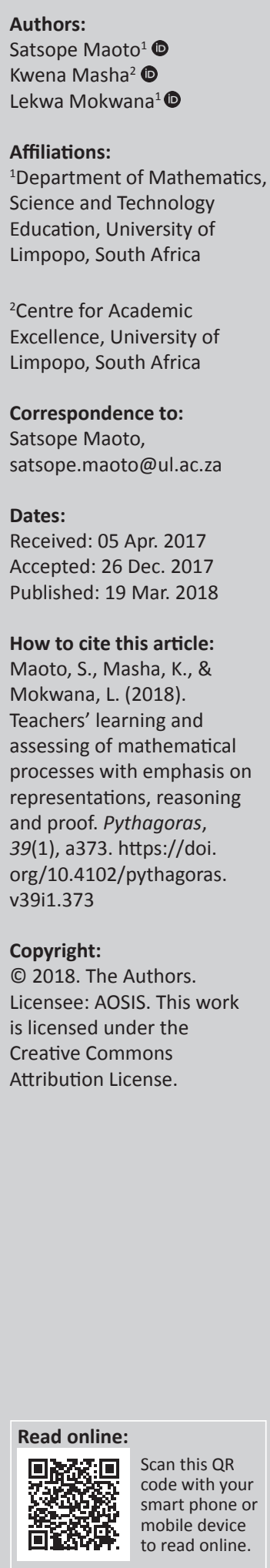

This article focuses mainly on two key mathematical processes (representation, and reasoning and proof). Firstly, we observed how teachers learn these processes and subsequently identify what and how to assess learners on the same processes. Secondly, we reviewed one teacher's attempt to facilitate the learning of the processes in his classroom. Two interrelated questions were pursued: 'what are the teachers' challenges in learning mathematical processes?' and 'in what ways are teachers' approaches to learning mathematical processes influencing how they assess their learners on the same processes?' A case study was undertaken involving 10 high school mathematics teachers who enrolled for an assessment module towards a Bachelor in Education Honours degree in mathematics education. We present an interpretive analysis of two sets of data. The first set consisted of the teachers' written responses to a pattern searching activity. The second set consisted of a mathematical discourse on matchstick patterns in a Grade 9 class. The overall finding was that teachers rush through forms of representation and focus more on manipulation of numerical representations with a view to deriving symbolic representation. Subsequently, this unidirectional approach limits the scope of assessment of mathematical processes. Interventions with regard to the enhancement of these complex processes should involve teachers' actual engagements in and reflections on similar learning.

\section{Introduction}

This article is based on a study aimed at the improvement of the quality of teaching and learning mathematics using authentic real-life mathematics explorations. This involves paying closer attention to relationships between the manipulation of concrete material and the arising numerical values. We particularly focus on the early stages of the study and use two mathematical processes: representation, and reasoning and proof (National Council of Teachers of Mathematics [NCTM], 2000), incorporating adaptive reasoning (Kilpatrick, Swafford \& Findell, 2001). The study addresses some of the expectations with regard to the same processes within the context of the South African Mathematics Curriculum and Assessment Policy Statement (Department of Basic Education, 2011). Our specific concern was how teachers' own learning of mathematical processes influence assessments of their learners. We limited the scope of this article by focusing on assessment of patterns arising from 2D and 3D shapes.

It has been a while since curricula developers and implementers strove for emphasis of both subject content and mathematical processes in mathematics teaching, learning and assessment (Kilpatrick et al., 2001; NCTM, 2000; Orton \& Frobisher, 1996). There are many processes to be experienced and developed. Orton and Frobisher (1996) classified general processes into four categories: communication, operational, recording, and reasoning, which they claimed could contribute to some mathematical processes. They argued that these mathematical processes are not unique to mathematics, but play an important role in the establishment of new ideas and structures within mathematics. The NCTM (2000) described problem-solving, reasoning and proof, communication, connections, and representations as process standards. Kilpatrick et al. (2001) proposed five intertwining strands of mathematical proficiency: conceptual understanding, procedural fluency, strategic competence, adaptive reasoning, and productive disposition. The development of mathematical proficiency takes time and it would be interesting to analyse teachers' assessments for process engagements.

It is recognised that, in addition to assessing content knowledge, assessments should provide insight into students' ability to engage with mathematical processes (Davis, Smith, Roy \& Bilgic, 2014; Gulkilik \& Arikan, 2012; Hunsader et al., 2014). Gulkilik and Arikan (2012) determined 
pre-service secondary mathematics teachers' views about using multiple representations in mathematics lessons. They found that, although the participants had concerns about the usage of multiple representations, they believed that using them was necessary for mathematics instruction. Davis et al. (2014) examined students' opportunities to engage in reasoning and proving within exposition and task components of two US reform-oriented secondary algebra textbooks. Hunsader et al. (2014) analysed the extent to which students have opportunities to engage in the processes within the tests accompanying published textbooks. They intended to support teachers' ability to make their own decisions about the efficacy of their assessment. Studies that investigate classroom instruction, or the actual assessments administered to students, continue to be important in order to support the development of specific pedagogical content knowledge. Because of this view, we were prompted to analyse 10 high school mathematics teachers' learning of mathematical processes and how to assess them. In particular, we focused on the design of appropriate assessment rubrics and the facilitation of learning that takes into account those expectations. It is important that teachers' values about assessment and their associated practices are consistent with each other and are both pedagogically sound.

\section{Theoretical framework}

This article is framed by the process standards as set out by the NCTM (2000) and Kilpatrick et al.'s (2001) theory of mathematics proficiency. The NCTM's process standards describe ways in which all students learn the algebra content by engaging in problem-solving, reasoning and proof, communication, making connections, and multiple representations. According to Kilpatrick et al.'s (2001) theory, mathematics proficiency comprises five intertwined strands: conceptual understanding, procedural fluency, strategic competency, adaptive reasoning, and productive disposition. While the bigger study utilises all these mathematical processes and strands, we focus on representation, reasoning and proof incorporating adaptive reasoning to analyse how teachers learn these processes and, subsequently, learn how to assess learners on the same processes. We analyse the teachers' learning from their written responses to a pattern searching activity.

The NCTM (2000) points out that representation refers both to process and to product. The emphasis is that students should have opportunities to view and to create multiple representations of mathematics graphically, numerically, algebraically, and verbally. Reasoning and proof should be a natural activity, an ongoing part of classroom discussions, no matter what mathematics topic is being studied. Particularly, 'students should expect to explain and justify their conclusions; ... to clarify their thinking, ... and to develop standards for high-quality mathematical reasoning' (NCTM, 2000 , p. 342). Meanwhile, Kilpatrick et al. (2001, p. 5) say that adaptive reasoning comprises the 'capacity for logical thought, reflection, explanation and justification'.

\section{Methodology}

We used case study research design to analyse 10 high school mathematics teachers' learning of representations, reasoning and proof as mathematical processes and how to assess them within the context of their university studies towards a Bachelor in Education Honours degree in mathematics education. Data constructed in this manner, where we could actually talk directly to participants and see them behave and act within their context and ultimately reflect on their own learning, are a major characteristic of qualitative research (Creswell, 2007). We used two sets of data. The first set of data comprised the teachers' written assessment task (Figure 1) submitted as part of the assessment requirements for a module that was delivered on a part-time basis. With the first six questions we took the teachers through a practical exercise that allowed them to learn, with understanding, the assessment of mathematical processes as expected in the other two questions.

The second set of data arose from the Grade 9 lesson activity of one of the teachers (Figure 2).

The class had 14 learners, 9 girls and 5 boys. They formed three groups: two of five members each and one with four members. This teacher's class was selected to provide a window through which to see how the participants' newly acquired knowledge and skills unfolded in an actual classroom environment.

Our analysis of the two sets of data was guided by the NCTM's (2000) process standards of representation, reasoning and proof, and Kilpatrick et al.'s (2001) notion of adaptive reasoning. For the first data set we drew our analysis from the teachers' responses to Item 5 and Item 8 (see Figure 1). For Item 8 we analysed the teachers' designed assessment criteria, targeting descriptions of the cells with

Pattern searching as a mathematical process - What and how to assess?

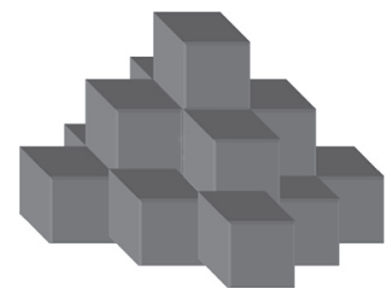

1. Find the number of cubes in the pyramid above.

2. Draw the next pyramid.

3. Find the number of cubes that are needed to build a pyramid that is 10 cubes high.

4. In simpler language, explain how you solved the questions above.

5. How many cubes will you need to build any pyramid similar to the one above?

6. Reflect on how you solved question 5 and indicate those critical issues/features/sub problems you had to resolve in the process.

7. What should be assessed in the given pyramid activity?

8. Design an assessment rubric for what you identified in question 7 .

FIGURE 1: Pyramid activity. 
Learning activity: Matchsticks pattern

1. Construct a series (chain) of matchstick squares and complete the table below:

\begin{tabular}{|l|l|l|l|l|l|}
\hline Number of squares & 1 & 2 & 3 & 4 & $n$ \\
\hline Number of matchsticks & & & & & \\
\hline
\end{tabular}

2. How many matchsticks do you need for the next square?

3. How many matchsticks will you need to construct 16,40 and 72 squares? Show all your calculations.

4. How many matchsticks will you need to construct any number of squares?

FIGURE 2: Matchsticks pattern activity.

the highest score to find out what and how they would assess. For the second data set, our analysis was guided by drawings that the learners produced in response to Item 4 and their associated conversations to explain and justify their conclusions.

\section{Ethical considerations}

This qualitative study complied with the university's ethical requirements. Approval was obtained from the relevant university structures and the 10 participants. The nature and purpose of the study were declared, inclusive of potential audiences and substantive foci. Erickson (1998) writes:

consent that is genuinely informed and without coercion reduces the risk of social harm because it affirms the dignity and respects the agency of those who will be involved in the study. (p. 1161)

We agreed on anonymity of the participants, hence no individual identities were divulged and pseudonyms were used. The teachers were beneficiaries of the study. They were inducted into the dynamics of what it means to learn and to assess mathematical processes with a view to teaching and assessing their own learners.

\section{Quality criteria}

Prolonged engagement (semester), persistent observation, ongoing probing during a number of whole-class discussions, peer debriefing and member checks provided sufficient opportunity to hear the teachers' voices, which contributed to establishing the credibility of this study (Bitsch, 2005; Guba \& Lincoln, 1989). Recursive discussions with the teachers and sufficient descriptive data added to the confirmability and transferability of this study (Guba \& Lincoln, 1989).

\section{Findings and discussions}

This section is organised according to the NCTM's (2000) process standards of representation, reasoning and proof. The latter incorporates Kilpatrick et al.'s (2001) notion of adaptive reasoning. Each of the two process standards is further organised in relation to the teachers' own learning of process skills and into assessment of their learners in this.

\section{Representation}

Representation as a process standard is attained when a learner can demonstrate the ability to:

- Create and use representations to organise, record, and communicate mathematical ideas.

- Select, apply, and translate among mathematical representations to solve problems.

- Use representations to model and interpret physical, social, and mathematical phenomena.

The activities that the participants engaged with in both data sets provided opportunities for exposure to all aspects of representation. The given pattern that the participants engaged with in the first data set is unique in the sense that the cubes were organised in a specific way to make the pyramid. The number of cubes that are required to construct any other pyramid can be found using a variety of methods. The pyramids can be dismantled and the individual cubes counted, cubes in a layer could be counted and subtotals added, the structure could be analysed and the counting done using the outcomes of that structural analysis, etcetera. The structural analysis of the pattern has the potential to lead to techniques that preserve mathematical ideas among the various representations. Only two methods became conspicuous from the teachers' responses: counting cubes per layer of the pyramid, and counting cubes per layer and adding the subtotals. In each of these approaches we analyse the teachers' own learning of the mathematics process of representation and their envisaged assessment of the process.

\section{Cubes per layer}

Seven respondents limited their calculations of the number of cubes in a particular layer of the pyramid. While the activity clearly asks for the number of cubes needed to build any pyramid similar to the one given, the participants interpreted that to require a number of cubes in any layer of a pyramid. Responses within this method also showed minor differences in terms of the additional techniques used.

The teachers' own learning in using only layers: These are how three of the seven teachers explained their work:

MKK: A general formula should be formulated that satisfies any number of cubes in each layer. I need a strategy to enable me to find the correct formula.

MAK: I extended the pyramid by extra layer.

(i) Write down the number of cubes in each layer of the pyramid.

(ii) Find the relationship between the number of cubes.

MLN: The first pyramid has 1 cube

the second $1+4=5$

the third $5+8=13$

the fourth $13+12=25$

The number of cubes in the previous pyramid plus the pyramid number times 4 gives the number of cubes 
in the next pyramid, i.e. for pyramid 3, the total cubes will be

$\mathrm{T}_{3}=2(3)^{2}+2(3)+1$

$=25$

The approaches fell short of addressing the total number of cubes required to build any pyramid. The numerical patterns generated from the approaches were a reflection of the pictorial patterns of the layers in a pyramid and not pictorial patterns of the pyramids themselves.

Once the numerical patterns were generated, the respondents applied three different techniques to arrive at the generalisation. The first technique (1 response) could be regarded as trial and error, as the generalisation was first presented and a few items were tried out. There is no clear evidence as to how such a generalisation was arrived at. For example, MLN had $\mathrm{T}_{n}=2 n^{2}+2 n+1$ as the general formula. MLN used $n-1$ to compute the value for $n$. The value that is calculated as $T_{3}$ is for the fourth pyramid. However, it is presented as the value for pyramid three. That is clearly a misrepresentation that defeats the purpose of harnessing this process skill.

With the second technique (2 responses), generalisation was arrived at through the use of 'met before' techniques of finding the differences between consecutive terms in the numerical patterns until a constant difference is found. It was MAK and MKK who used the technique, arriving at the conclusion that $\mathrm{T}_{n}=2 n^{2}-2 n+1$. While this generalisation is a true generalisation of the numerical pattern of the number of cubes in a layer, this representation has no structural relevance to the pictorial representation of the layer. The manipulation of the numerical patterns did not mimic the concrete manipulation of the pictorial representation of the layers.

Using the third technique (2 responses), generalisation was also given as $\mathrm{T}_{n}=2 n^{2}-2 n+1$. This outcome emanates from a different approach in analysing the numerical pattern. Each term in the sequence is seen as a sum of two consecutive squares - the square of $n$ and that of $(n-1)$. STT and MMW provide examples of how that was arrived at.

STT: Make a sequence of squaring the natural numbers: $1 ; 4 ; 9 ; 16 ; 25$ etc. Add the square numbers starting from the second term (e.g. $4+1 ; 9+4 ; \ldots)$. This means I squared the level number and add level plus one squared (e.g. $\left.1^{2}+(1-1)^{2}=1 ; 2^{2}+(2-1)^{2}=5\right)$.

\begin{tabular}{l|l|l|l|r|r|l|l|l|} 
MMW: & Independent & 1 & 2 & 3 & 4 & $\ldots$ & 10 & $n$ \\
\hline Dependent & 1 & 5 & 13 & 25 & $\ldots$ & $?$ & \\
\hline
\end{tabular}

[Here MMW shows how entries in the table were calculated.]

$$
\begin{array}{ccccc}
1^{2} & 2^{2} & 3^{2} & 4^{2} & 5^{2} \\
1 & 4 & 9 & 16 & 25 \\
+1 & +4 & +9 & +16 \\
L^{2}+(L-1)^{2}= & L^{2}+L^{2}-2 L+1=2 L^{2}-2 L+1
\end{array}
$$

The technique deployed by both STT and MMW can be regarded as sums of two consecutive squares. Expressing the generalisation in this way allows for a closer review of the pictorial representations of the layers of the pyramid with a view of determining what that translates into. The model allows for the interpretation of physical mathematical phenomena, a core aspect of representation.

The last two participants of the seven (MST and KAS) in this category had insufficient responses for what the activity had asked for. However, the opportunity to learn about the processes was not adversely affected. The issue is the depth of that learning and how that was translated into the designed assessment criteria in the context of representation.

The teachers' approach to assessment of learning: The focus in this section falls on five of the seven participants whose responses were regarded as sufficient. While MLN had a completely irrelevant response (no connection between the response and Item 5), the other four teachers had similar responses. Two issues were targeted for assessment and those were the ability to recognise the pattern and the ability to generalise the pattern. Furthermore, the expectation of the four teachers in terms of the two criteria closely resembles how they dealt with the problem in Item 5 . Here are how the four teachers expressed performance at the highest level in relation to the two criteria.

MKK: Able to recognise observable, hidden and underneath cubes to extend the pattern Show outstanding understanding of relating number of cubes and layers

STT: Ability to recognise cubes observable and hidden Show a complete understanding

MAK: All occurrences mentioned and logically done Create a pattern using the observable, behind and underneath cubes and able to generalise the pattern

MMW: Ability to:

- recognise cubes, hidden and those underneath and could extend the pattern

- derive a formula

The responses were, to a limited extent, in line with one of the three aspects of representation: 'create and use representations to organise, record, and communicate mathematical ideas'. The limitation of the responses is in relation to the amount of time spent on the manipulation of the way the layers are structured. In responding to Item 5, these teachers were content with the numbers without checking whether those numbers adequately communicated the structure of the layers. The drawing of the structures of the layers, analysing the structures, and subsequently recording the observations are the actions that make representation a process standard. However, there is no clear evidence that an in-depth attempt was made in that regard. MAK's expectation that all occurrences should be mentioned and logically done can be tricky if considered out of context. At the lower levels he expects one, two, or three occurrences, while remaining unclear about what these occurrences are. However, if one reflects back on MAK's own engagement with the activity, these occurrences could be the different layers of the pyramid. 
The responses in relation to generalisation also show the participants' obsession with symbolic representation that has no direct relation with the structural aspects of what is represented. It is not immediately clear what MKK and STT refer to by understanding, but that could be in line with what MMW regards as a formula that will help find the number of cubes per layer. Approached in this way, the opportunity for translation among mathematical representations is very limited. Again, the appreciation of the generalisation as a model for the pyramids is lost and therefore the opportunity to fully develop representation as a process standard is limited.

\section{Cubes per layer and the totals for the pyramid}

Three participants fell in this category. They first calculated the number of cubes per layer and then added the subtotals to find the required number of cubes for any pyramid.

The teachers' own learning in using layers and pyramid totals: All three participants arrived at the generalisation of $T_{n}=\frac{2}{3}\left(n^{3}\right)+\frac{1}{3}(n)$. The conclusion was reached using similar approaches, with MLL hinting at an additional strategy.

CKG: Consider the number of layers per pyramid and number of cubes per layer. Number of cubes per pyramid is:

$1 ;(1+5=6) ;(1+5+13=19) ;(1+5+13+25=44) ;$ $(1+5+13+25+41=85)$

$1 ; 6 ; 19 ; 44 ; 85 ; \ldots$

First difference: $5 ; 13 ; 25 ; 41$ - not constant

Second difference: $8 ; 12 ; 16 ; 20$ - not constant

Third difference: $12-8=4 ; 16-12=4 ; 20-16=4$ is constant, then the pattern is cubic

HMJ: Write the pattern. Find the differences ... first differences not common means - not a linear pattern, second differences not common - is not a quadratic pattern, the third difference is common therefore is a cubic pattern. First difference $=5 ; 13 ; 25 ; 41 ; 61 ; 85 ; 113 ; 145 ; 181$ Second difference $=8 ; 12 ; 16 ; 20 ; 24 ; 28 ; 32 ; 36$ Third difference $=4$

MLL: I used the conjecture I formulated in question 3 as basis for generalisation. Number of cubes per layer form a quadratic pattern, thus the rule is of the second degree. Squared the term number and multiplied it by two, subtracted different numbers for different layers. Different numbers I subtracted formed a linear pattern which enabled me to generalise the number of cubes per layer. Alternatively:

Total number of cubes needed to build pyramids with layers 1 to 5 will be $1 ; 6 ; 19 ; 44 ; 85$.

Differences between consecutive terms:

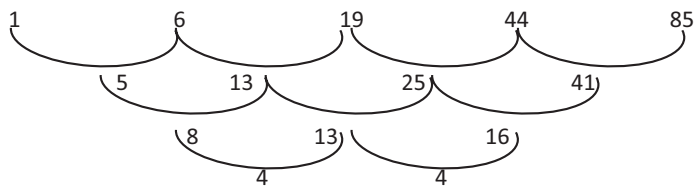

From prior knowledge, a pattern with a constant difference yields a linear pattern, and with second constant difference yields a quadratic pattern. The one with a third constant difference yields a cubic pattern:
$\mathrm{T}_{n}=a n^{3}+b n^{2}+c n+d$, the challenge is to find the values of $a, b, c$, and $d$.

The three participants clearly worked towards establishing the number of cubes in a pyramid. Counting the cubes in each layer was considered as a step in that process. The need for justification for manipulation of the numerical patterns is evident in the responses. The implication of those manipulations on the structure of the layers is not, however, reflected in the way the subtotals were found, especially in CKG's and HMJ's responses. MLL hinted at an attempt to analyse the structure of the layers without concluding that aspect. Meanwhile, all the three responses show that once the numerical sequence was established, the general strategy of determining the differences among consecutive terms was used to arrive at a conclusion that the general pattern is a cubic one. That strategy was then correctly used to arrive at the generalisation of $T_{n}=\frac{2}{3}\left(n^{3}\right)+\frac{1}{3}(n)$.

The teachers' approach to assessment of learning: The three participants, while they generally highlighted both the ability to generate a pattern and generalise it, placed different emphasis on the two aspects. The expected performance at the highest levels in relation to the given criteria was as follows:

CKG: Correctly count front, behind, hidden cubes and correctly draw next pyramid Correct manipulation of numbers, variables and operations and make a relevant conclusion

HMJ: Use front, back and underneath to extend pattern Able to generalise correctly, logically and appropriately

MLL: Total cubes per layer and per pyramid are correct. Correct description of the patterns in words or using conjectures or any mathematical calculation, for both cubes per layer and per pyramid Correct generalisation for both the number of cubes per layer and per pyramid

It was clear in all the responses that there was more than generalisation that was expected. HMJ wanted to see the logic, CKG wanted to see the manipulations of numbers and variables and the relevance of that in the conclusion, while MLL wanted descriptions or conjectures for both the layers and the totals. If strictly and consistently applied, these assessment rubrics could encourage the realisation of the core aspects of representation. However, in their current format, it is not clear that navigation between multiple representations is being encouraged. It is only when we take a look at how MLL facilitated learning in his classroom that this aspect became clearer.

\section{MLL's facilitation of the matchsticks activity}

In engaging with the assigned activity to construct a series of chained squares, their associated number patterns and the generalisation thereof, three groups of learners in MLL's classrooms used different approaches and representations. Initially, two patterns were generated. However, at the end there were three as a result of the amendment of one of them. Three distinct representations from two groups of learners 
were observed from data set 2 . The associated conversations also articulate the aspects of representation.

The two groups presented these drawings on the board. This was the drawing for Group 1:

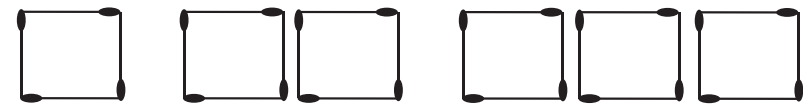

This was the drawing for Group 2 and Group 3:
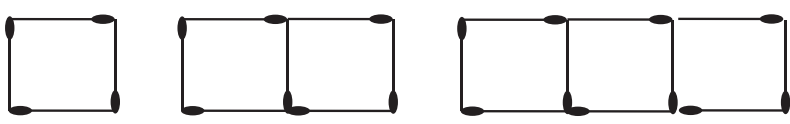

Jahi: [Group 1] Each square required four matchsticks and for every additional square four matchsticks were required.

16 squares: $16 \times 4=64$ matchsticks

40 squares: $40 \times 4=160$ matchsticks

72 squares: $72 \times 4=288$ matchsticks

Any number of squares $x \times 4$

Jahi: [Saw how other learners frowned at the presentation, turned to one of them.] Bongi, what's wrong?

Bongi: Are your drawings forming a chain?

Jahi: [Looked at the drawings, turned to Bongi and then at her group silently asking for assistance.]

Kabo: [From Group 1 walking towards the board.] Oh, a chain, they must be joined together. But it will not make a difference on our answer. [Redrew this amended drawing.]
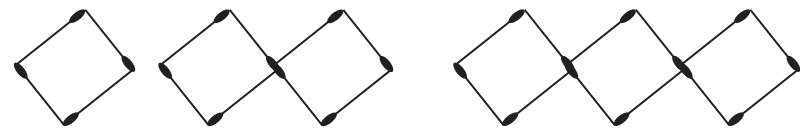

Group 2 and Group 3 had the same series of matchstick squares, with same pattern and conjectures. However, Group 3 could not generalise the pattern and Group 2's generalisation did not show any relationship with their conjecture.

Thalan presented Group 2's responses.

Thalan: The first square required four matchsticks and for every additional square, three match-sticks were required.

16 squares: $4+3 \times 15=49$ match-sticks

40 squares: $4+3 \times 39=121$ match-sticks

72 squares: $4+3 \times 71=217$ match-sticks

Any number of squares: $x \times 3+1$

[He was questioned why the 4 disappeared in the generalisation and where the 1 came from. This had an effect on the group as Thalan changed the presentation and wrote for 16 squares: $16 \times 3+1=49]$

Rof: [from Group 3] I have a different general rule. For 16 squares, they needed $4+3 \times 15=49$ matchsticks and 15 which is multiplied by 3 is one less than 16 . So the general rule is $4+3(x-1)$ [Wrote it on the board]. Therefore, for any number $x$ there will be $4+3(x-1)$ matchsticks.

The learners agreed with Rof that his general rule was working and was observable in the conjecture. But the question raised was why there were two different generalisations that are both working. It was at this stage that the teacher, MLL, asked the learners to simplify the expression and see what would happen. There was much excitement when the expression reduced to the other group's general rule of $x \times 3+1$.

In MLL's classroom activity, the learners' descriptions, drawings, their general rules and numerical representations were all being interrogated with a view to establish direct connections among them. The 4 in the general formula was expected to be in the numerical representation (conjecture) and in the actual drawings. The learners were not only interested in the final general rule but clear translation among the different representations. The elements of the omnidirectional value in engaging with representations as a mathematics process were becoming evident. These kinds of learning interactions that we observed in MLL's class take time to inculcate. The classroom learning environment allowed for demonstration of knowledge and skills commensurate with MLL's assessment criteria (recognition, description in words or using conjectures or any mathematical calculation, and generalisation of the number pattern).

\section{Reflective thoughts on representation}

At this stage of the study, we observed that, when faced with an authentic mathematical problem that requires different facets of representation, teachers tend to rush into generation of numerical representations. This stage is then followed by manipulations with a view to deriving symbolic representations. The process is almost exclusively unidirectional and, thereby, limits the opportunity for the richness of each representation's contribution in developing insights into other representations.

\section{Reasoning and proof}

Reasoning and proof as a mathematics process standard requires of learners to:

- recognise reasoning and proof as fundamental aspects of mathematics

- make and investigate mathematical conjectures

- develop and evaluate mathematical arguments and proofs

- select and use various types of reasoning and methods of proof.

During reasoning and proof, viewed from adaptive reasoning as a strand of mathematical proficiency, learners should develop capacity for logical thought, explanation, and justification. All of these traits were observable in almost all the responses in both data sets, although to varying degrees. The activity itself was designed to facilitate the learning of this process strand. For the data set that involved teachers (see Figure 1), Item 4 required that they explain how Item 3 was resolved while Item 6 required critical reflection on how Item 5 was resolved. The responses to the latter item were used earlier. For the current process standard, we use responses to Item 3 and Item 4 . 
One of the 10 participants (MST) gave a very brief response to the two activity items indicating that he used number series to arrive at an answer of 186 (total number of cubes). While 186 is incorrect, the lack of richer explanation made it difficult to establish his thought process. The responses of the other nine participants fell into three categories, viz. numerical approaches, numerical and symbolic approaches, and numerical, symbolic and pictorial approaches.

\section{Numerical approaches}

The numerical perspective of mathematics is very powerful as it constitutes the elementary nature of the discipline. Our interest at this point is how the participants' own learning, which is dominated by numerical approaches, translates into the assessment of learners.

Teachers' own learning in using numerical approaches: There were five participants who used a numerical approach. They used the existence of the numerical pattern as a justification for their response to Item 3. The strategy involved the establishment of the total number of cubes per layer, and subsequently adding those numbers to find the total for the pyramid. MKK's and STT's responses are used to demonstrate the strategy.

MKK

3. 670 cubes

Identify the number of observable and hidden cubes in each layer.

\begin{tabular}{|l|c|c|c|c|c|c|c|}
\hline No. of layers & 1 & 2 & 3 & 4 & $\ldots$ & 9 & 10 \\
\hline Cubes/layer & 1 & 5 & 13 & 25 & $\ldots$ & 145 & 181 \\
\hline Total cubes per pyramid & 1 & 6 & 19 & 44 & $\ldots$ & 489 & 670 \\
\hline
\end{tabular}

Alternatively:

$1^{2}=1$

$2^{2}=4+1^{2}=5$

$3^{2}=9+2^{2}=13$

$4^{2}=16+3^{2}=25$, etc.

STT

3. The number of cubes needed to build a pyramid that is 10 cubes high is 670 .

\begin{tabular}{|c|c|c|}
\hline Level & Cubes/level & Total cubes/pyramid \\
\hline 1 & 1 & 1 \\
\hline 2 & 5 & 6 \\
\hline 3 & 13 & 19 \\
\hline$\vdots$ & $\vdots$ & $\vdots$ \\
\hline 10 & 181 & 670 \\
\hline
\end{tabular}

For the total cubes per pyramid (e.g. $1+5=6,1+5+13=19$ ) I calculated the differences between successive terms and the common difference was found in the second difference. I used the common difference to generate the sequence for the number of cubes per level. I cumulated the generated pattern to get the total cubes for pyramid that is 10 levels high.

Both MKK and STT went into detail to demonstrate how they arrived at their answer to Item 3. That, on its own, demonstrated that the participants do value reasoning and proof as important aspects of mathematics. It is evident that more work was also done elsewhere before the results were captured in their tables. The first step of their response was to generate the number of cubes per layer and later add those to get the number for the pyramid. That is, the participants established and reasoned for their response by actually calculating all the previous sums until the 10th. The power of numerical pattern was preferred. The only structural property of the pictorial pattern that was used is the layered nature of the pyramid. The structure of the layers themselves did not feature. We also observed that MKK did pursue an alternative approach to establish the answer; however, that was also numerical. While both participants achieved their goal of determining the actual number of cubes required to build the required pattern, their limited focus on numerical approach constrains the opportunity of encountering the varied ways through which the answer can be established. The critical aspect of mathematics is not its ability to resolve a problem but its broadness in facilitating such a resolution. Justifying and proving the solution of the given problem using a single algebraic strategy of numerical pattern is less convincing as it falls short of room for a selection and use of various types of reasoning and methods of proof.

The teachers' approach to assessment of learning: Indicators on what the participants valued in relation to assessment of learning are derived from their responses to Item 7 and Item 8 of the given activity. We again use MKK's and STT's responses. They both identified similar skills to be assessed with one difference.

\section{Ability to:}

- generate a number pattern.

- sequentially/logically record data.

- find relationship of layer and total cubes per layer.

- give a convincing explanation in a narrative/mathematical form.

The only difference was that MKK included 'interpretation of word problems' while STT had 'ability to generalise'. As was observed with their own learning, the two participants value the generation of number pattern and relating that to layers and total number of cubes. The reasoning and proof is limited to 'giving convincing explanation'. While this acknowledges the significance of reasoning and proof, it falls short of encouraging the varied nature of how that could be undertaken.

\section{Numerical and symbolic approaches}

By numerical and symbolic approaches, we refer to a scenario in which the strategy is considered credible when a symbolic generalisation is established. A numerical pattern is generated and analysed with a view to generalise it and only then is it applied to resolve prevailing problems.

Teachers' own learning in using numerical and symbolic approaches: There were three participants in this category. In addition to what the participants in the previous category 
did, this group went further to establish the generalisation for number of cubes per level and in other cases also for the pyramid. The generalisability of the number of cubes was considered reason enough to accept the response to Item 3. We use KAS's and MLL's responses to demonstrate the strategy.

KAS
\begin{tabular}{|l|c|c|c|c|c|c|c|c|c|c|}
\hline No. of layers & 1 & 2 & 3 & 4 & 5 & 6 & 7 & 8 & 9 & 10 \\
\hline $\begin{array}{l}\text { No. of cubes } \\
\text { per layer }\end{array}$ & 1 & 5 & 13 & 25 & 41 & 61 & 85 & 113 & 145 & 181 \\
\hline $\begin{array}{l}\text { Total cubes } \\
\text { per pyramid }\end{array}$ & 1 & 6 & 19 & 44 & 85 & 146 & 231 & 344 & 489 & 670 \\
\hline
\end{tabular}

$$
\begin{gathered}
\mathrm{T}_{1}=1^{2} ; \quad \mathrm{T}_{2}=2^{2}+1 ; \quad \mathrm{T}_{3}=3^{2}+4 ; \quad \mathrm{T}_{4}=4^{2}+9 ; \quad \mathrm{T}_{5}=5^{2}+16 ; \\
\mathrm{T}_{10}=10^{2}+81=100+81=181
\end{gathered}
$$

Square the number of layer and add the square of the previous number of layer.

\begin{tabular}{|c|c|c|}
\hline \begin{tabular}{c|}
$\begin{array}{c}\text { Number of } \\
\text { layer }\end{array}$ \\
\end{tabular} & Cubes/layer & Total \\
\hline 1 & $\begin{aligned} 1 & =2(1)^{2}-1 \\
& =2(1)^{2}-2(1)+1\end{aligned}$ & $1=\frac{2}{3}(1)^{3}+\frac{1}{3}(1)$ \\
\hline 2 & $\begin{aligned} 5 & =2(2)^{2}-3 \\
& =2(2)^{2}-2(2)+1\end{aligned}$ & $6=\frac{2}{3}(2)^{3}+\frac{1}{3}(2)$ \\
\hline 3 & $\begin{aligned} 13 & =2(3)^{2}-5 \\
& =2(3)^{2}-2(3)+1\end{aligned}$ & $19=\frac{2}{3}(3)^{3}+\frac{1}{3}(3)$ \\
\hline 4 & $\begin{aligned} 25 & =2(4)^{2}-7 \\
& =2(4)^{2}-2(4)+1\end{aligned}$ & $44=\frac{2}{3}(4)^{3}+\frac{1}{3}(4)$ \\
\hline 10 & $2(10)^{2}-2(10)+1=181$ & $\frac{2}{3}(10)^{3}+\frac{1}{3}(10)=670$ \\
\hline
\end{tabular}

MLL

I created a conjecture that relates the number of layers, the numbers of cubes per layer and per pyramid. The conjecture holds for the first 5 terms of the pattern, hence I applied it to a pyramid 10 layers high.

In KAS's case, the generalisation was with regard to the number of cubes per layer. While the origin of the idea is not clear, KAS used the sum of squares to arrive at the number of cubes per layer. The number of cubes for a pyramid is not problematised as it is the sum of cubes per layer. MLL worked with a conjecture for both the layers and the pyramid. Once it was established that the conjecture worked for a few items in the pattern, the idea was extended to establish the answer for Item 3.

The three participants in this group demonstrated their appreciation of reason and proof as valuable aspects of mathematics. The need to establish and test the conjectures was attempted which made their responses qualitatively different from those in the first category. However, more still needs to be done to address other aspects of reasoning and proof as the process standard requires. The multiplicity of methods of proof still need to be demonstrated. It is in the category that follows where the emergence of the idea is observable.
The teachers' approach to assessment of learning: For the sake of continuity, KAS's and MLL's responses are used.

KAS: Skills to assess:

- Sequential/logical recording of data

- Ability to relate numbers

- Interpretation

- Generalisation, and

- Substitution

MLL: Could assess ability to:

- count the number of cubes per layer and per pyramid.

- recognise a number pattern from the number of cubes per layer and per pyramid.

- extend the number pattern by both drawing and working with the pattern recognised.

- describe the pattern recognised on cubes per layer and per pyramid.

- generalise the described pattern for any pyramid.

- verify the generalised rule for the patterns.

Evident in the two responses is that generalisation is not seen as an end in itself. There is clear expectation that the learning should include the application of the generalisation. Furthermore, MLL expects the ability to carry out the extensions not only numerically but also pictorially. The latter requires exploration and understanding of the structural aspects of the pictorial patterns. What remains unclear, however, is the ability of learners to relate structural properties of the pyramids with the numerical or symbolic patterns. This shortfall limits the potential for meaningful interpretation of the various strategies that can be used to address the problem.

\section{Numerical, symbolic, and pictorial approach}

Approaching learning from a variety of perspectives has a potential for triangulation, deeper and meaningful learning. In many respects, this is closer to the ideals of reasoning and proof as a mathematical process.

Teacher's own learning with respect to numerical, symbolic and pictorial approach: Only one participant, MLN, unpacked and analysed the pictorial pattern and thus attempted to relate the structural properties of the layers and their numerical patterns. This is an important aspect in the development of reasoning and proof as a mathematics process standard. Analysis of pictorial patterns allows for meaning making and has the potential for multiplicity of reasoning and methods of proof.

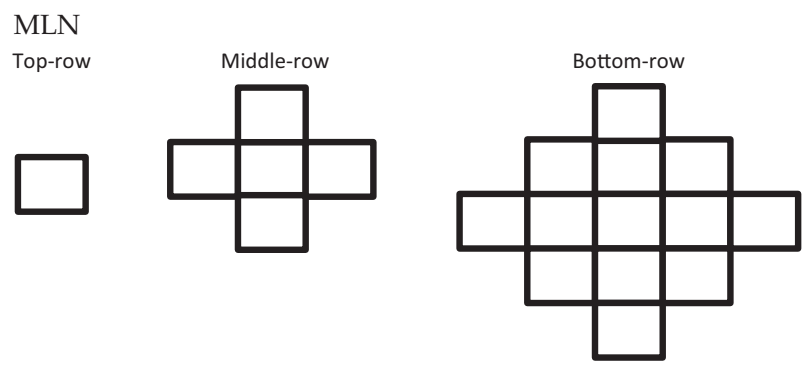


3.

$$
\begin{aligned}
& 1^{\text {st }} \text { top row }=1 \text { cube } \\
& 2^{\text {nd }} \text { top row }=5 \text { cubes } \\
& 3^{\text {rd }} \text { top row }=13 \text { cubes } \\
& \vdots \\
& 10^{\text {th }} \text { top row }=181 \text { cubes }
\end{aligned}
$$

Number of cubes needed to build a pyramid that is 10 cubes high is:

$1+5+13+25+41+61+85+113+145+181=670$

5. The number of cubes in the given pyramid was obtained by first drawing the view of each row of the pyramid and then add them.... Number of cubes for a pyramid 10 cubes high was obtained by counting the number of cubes per row and then find the sum.

In this response it is clear how the numbers of cubes per layer were obtained. The relationship between the numerical and the pictorial patterns is clearer as compared to the previous cases. To an extent, the explanation is more convincing as the reorientation of the pictorial pattern reveals the hidden cubes. As an attempt to justify the response to the activity Item 3, MLN's presentation had fewer uncertainties as both the pictorial and numerical patterns complemented each other. This double method of representation advanced the opportunity to acquire reasoning and proof as a mathematics process standard.

Teacher's approach to assessment of learning: MLN's approach to assessment was distinct as compared to other participants. Instead of the usual expectation of generating a pattern and ability to generalise, she emphasised geometric and problem-solving skills. This is how she responded to the task list of issues that should be considered for assessment.

MLN: Skills to assess

- Drawing skills: Understanding how to draw the figure and the actual drawing skill

- Arrangements: Area models and set models

- Counting: Use of patterns in counting

- Problem-solving: Understanding of the problem; taking risks; and justifying results

There is an obvious attempt to integrate geometric and algebraic skills in the assessment. The inclusion of problemsolving skills also provides for further opportunities in which individual learners could bring additional skills into the problem. If properly applied, then this approach has the potential to encompass all the attributes of 'reasoning and proof'. MLN's invested energies in engaging with the given activity congruently translated into his expectations of what the learners should be able to do.

\section{Reflective thoughts on reasoning and proof}

The development of attributes for reasoning and proof require a culture of multiplicity in the resolution of problems. Both teachers and learners need to appreciate that it is not necessarily the final answer that is essential but the variety of ways of accomplishing the task matters. We have observed that while the participants demonstrated an appreciation for reasoning and proof, the preoccupation with the final answer limited explorations of other forms of proofs. The practice translates into limited scope of assessment.

\section{Conclusion and implications}

Mathematics process standards, as covered in this study, are broad and long term in nature. In engaging with them we acknowledge the need to take into account the bigger picture (Maoto, Masha, \& Maphutha, 2016) that underpins each of those standards. The learning activities presented as part of this article were not meant to address these mathematical processes fully, but to set in motion a concerted effort to expose the participants to such mathematics processes. In closing, we revert back to the questions raised in the study.

\section{Teachers' challenges in learning mathematical processes}

The presentation of the pictorial pattern that was used to engage the teachers offered multiple scenarios of engagement in the process of deriving symbolic representations. In addition to the various forms of experiences and interpretations that individual participants brought to the activity, the outcomes of the engagements were plenty. However, what became evident is the challenge of rushing the process towards numerical and symbolic representations. Once the numbers were derived from the pictorial representation, different levels of manipulation of the numbers became the main focus away from the structural aspects of the original figural pattern. For instance, while the activity item clearly asks for the number of cubes needed to build any pyramid similar to the one given, we observed 7 of the 10 participants interpreting and limiting themselves to calculating the number of cubes in a particular layer of the pyramid. This bottleneck in the generated pattern might be due to the perception of mathematics as being about numbers. The unidirectional approach that they used made it impossible for them to realise the discrepancies in their responses. Meanwhile, the other three participants remained focused and continued to find the totals for the pyramid as required; they missed the bigger picture in resolving the problem due to their confinement to numerical and symbolic patterns.

Justifying and proving a solution through a single strategy lacks rigour that is required for one to appreciate reasoning and proof as a mathematics process. In our case the function type determined by the non-linear nature of the task challenged the participants' reasoning capabilities. While it was easier for them to generalise a linear pattern (generated from counting the cubes per layer), they had difficulty in generalising what emerged as a non-linear pattern. This confirms Jurdak and Mouhayar's (2014) view that the development of reasoning in pattern generalisation is mainly due to experience rather than maturation. The slight moderation of MLN's attempt to integrate the pictorial and numerical approaches better presented a double method of representation that complemented each other and thus advanced the opportunity to acquire reasoning and proof as a mathematics process standard (see Figure 3). 


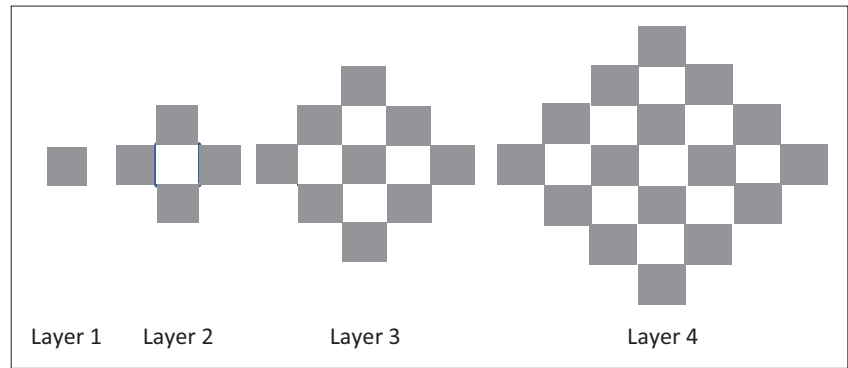

FIGURE 3: Pyramid layers with cubes coloured differently for emphasis.

MLN's unpacking and analysis of the pictorial pattern provided her an opportunity to develop capacity for logical thought, explanation and justification. Investing more time in manipulating the different forms of representations, especially the pictorial or concrete representations, does contribute to richer insights into the ideas and concepts at play. Justification of the strategy is in its consistency of success in resolving a problem. While this provides some comfort, it is still not a replacement for a corroboration that is arrived at through varied forms of reasoning and proof. Authentic learning of mathematical processes requires that all representations be equally explored as that would lead to a smooth omnidirectional approach to learning. That kind of learning could be facilitated by teachers who have excellent translation abilities among multiple representations so that they could assist their learners to access and to comprehend abstract mathematical ideas.

\section{Teachers' learning and assessing of mathematical processes}

To some extent, we observed that when complex knowledge and skills are involved, teachers' own approaches to learning do reflect depth of their expectations of what would be learned. It was MLL and MLN who presented relatively better quality engagements in their learning and that led to qualitatively better expectations from their learners. However, what we observed from MLL's actual implementation of his newly acquired skills in a classroom offers some hope. Learners' ways of engaging with the activity offered more and better opportunities to acquire representation as a process skill. Links among different representations were being interrogated much more than MLL did in his own learning. The manipulatives (matchsticks) enhanced learners' learning as they interrogated and established connections among their multiple representations. This aligns to Uribe-Flórez and Wilkins's (2017) findings that the use of manipulatives encourages multiple representations and has a long-term effect on students' learning as opposed to immediate achievement. We observed the value of an omnidirectional approach to the development of mathematics processes. More importantly, it emerged that meaningful assessment is learned in the context of the concepts that should be assessed. Deeper understanding of the concept opens possibilities for varied and meaningful assessment. Interventions with regard to the enhancement of these complex processes should involve teachers' actual engagements in and reflections on similar learning.

\section{Acknowledgements Competing interests}

The authors declare that they have no financial or personal relationships that may have inappropriately influenced them in writing this article.

\section{Authors' contributions}

S.M. was the project leader and facilitated lessons for the assessment module towards a Bachelor in Education Honours degree in mathematics education at the university. L.M. collected and analysed data for the second data set. S.M. conceptualised the article and wrote the draft manuscript. K.M. played a role of a critical reader, improved on the logical flow of ideas and filled in the gaps that existed, thus improving the quality of the article. S.M. and K.M. then engaged in reconceptualising and critically analysed the two data sets to produce the final draft of the paper.

\section{References}

Bitsch, V. (2005). Qualitative research: A grounded theory example and evaluation criteria. Journal of Agribusiness, 23(1), 75-91. Available from http://purl.umn. edu/59612

Creswell, J.W. (2007). Qualitative inquiry and research design: Choosing among five approaches. (2nd edn.). Thousand Oaks, CA: Sage Publications.

Davis, J.D., Smith, D.O., Roy, A.R., \& Bilgic, Y.K. (2014). Reasoning-and-proving in algebra: The case of two reform-oriented U.S. textbooks. International Journal of Educational Research, 64, 92-106. https://doi.org/10.1016/j.ijer.2013.06.012

Department of Basic Education. (2011). Curriculum and assessment policy statement: Mathematics. Further Education and Training Phase. Grades 10-12. Pretoria: DBE.

Erickson, F. (1998). Qualitative research methods for science education. In B.F. Fraser \& K.G. Tobin (Eds.), International handbook of science education (pp. 1155-1173). Dordrecht: Kluwer.

Guba, E.G., \& Lincoln, Y.S. (1989). Fourth generation evaluation. Newbury Park, CA: Sage Publications.

Gulkilik, H., \& Arikan, A. (2012). Preservice secondary mathematics teachers' views about using multiple representations in mathematics instruction. Procedia-Social and Behavioral Sciences, 47, 1751-1756. https://doi.org/10.1016/j.sbspro. 2012.06.895

Hunsader, P.D., Thompson, D.R., Zorin, B., Mohn, A.L., Zakrzewski, J., Karadeniz, I., et al. (2014). Assessments accompanying published textbooks: The extent to which mathematical processes are evident. ZDM: The International Journal on Mathematics Education, 46(5), 797-813. https://doi.org/10.1007/s11858-0140570-6

Jurdak, M.E., \& El Mouhayar, R.R. (2014). Trends in the development of student level of reasoning in pattern generalization tasks across grade level. Educational Studies in Mathematics, 85(1), 75-92. https://doi.org/10.1007/s10649-013-9494-2

Kilpatrick, J., Swafford, J., \& Findell, B. (Eds.). (2001). Adding it up: Helping children learn mathematics. Washington, DC: National Academies Press.

Maoto, S., Masha, K., \& Maphutha, K. (2016). Where is the bigger picture in the teaching and learning of mathematics? Pythagoras, 37(1), a338. https://doi. org/10.4102/pythagoras.v37i1.338

National Council of Teachers of Mathematics. (2000). Principles and standards for school mathematics. Reston, VA: NCTM.

Orton, A., \& Frobisher, L. (1996) Insights into teaching mathematics. London: Cassell.

Uribe-Flórez, L.J., \& Wilkins, J.L. (2017). Manipulative use and elementary school students' mathematics learning. International Journal of Science and Mathematics Education, 15(8), 1541-1557. https://doi.org/10.1007/s10763-016-9757-3 